\title{
Electron neutrino tagging through tertiary lepton detection
}

\author{
L. Ludovici ${ }^{1}$, F. Terranova ${ }^{2, a}$ \\ ${ }^{1}$ I.N.F.N., Sezione di Roma Sapienza, Rome, Italy \\ ${ }^{2}$ I.N.F.N., Laboratori Nazionali di Frascati, Frascati (Rome), Italy
}

Received: 21 April 2010 / Revised: 13 June 2010 / Published online: 11 August 2010

(C) The Author(s) 2010. This article is published with open access at Springerlink.com

\begin{abstract}
We discuss an experimental technique aimed at tagging electron neutrinos in multi-GeV artificial sources on an event-by-event basis. It exploits in a novel manner calorimetric and tracking technologies developed in the framework of the LHC experiments and of rare kaon decay searches. The setup is suited for slow-extraction, moderate power beams and it is based on an instrumented decay tunnel equipped with tagging units that intercept secondary and tertiary leptons from the bulk of undecayed $\pi^{+}$ and protons. We show that the taggers are able to reduce the $v_{e}$ contamination originating from $K_{e 3}$ decays by about one order of magnitude. Only a limited suppression $(\sim 60 \%)$ is achieved for $v_{e}$ produced by the decay-in-flight of muons; for low beam powers, similar performance as for $K_{e 3}$ can be reached supplementing the tagging system with an instrumented beam dump.
\end{abstract}

\section{Introduction}

The identification of the initial flavour of neutrinos produced by artificial sources through the detection of the associated lepton ("neutrino tagging") is a possibility that has been envisaged many decades ago [1,2]. Its realization, however, must overcome major experimental challenges and, in spite of numerous proposals [3-8], a facility operating with $v_{e}$ or $v_{\mu}$ neutrinos tagged on an event-by-event basis is still to come. In a tagged neutrino facility, a precise knowledge of the neutrino flavour at the source can be achieved identifying the associated lepton in coincidence with the occurrence of a neutrino interaction at the far detector. As it will be shown in the following, the exploitation of this correlation requires time resolutions below $1 \mathrm{~ns}$ both for the taggers and for the neutrino detectors. In the past, two approaches have been pursued. The former — dating back to 1969 [1]—is targeted to the identification of $v_{\mu}$ from kaon decay: it takes advantage of the large difference in Q-value between $\pi$ and

a e-mail: francesco.terranova@cern.ch
$K$ decays to isolate leptons from kaons without intercepting muons from $\pi$ or the undecayed parent mesons. The second approach focuses on the identification of positrons in order to either select a pure $v_{e}$ beam for physics measurements $[6,7]$ or to veto the $v_{e}$ contamination in $v_{\mu}$ beams from $\pi$ decay-in-flight ("anti-tagging" [8]). The technique that we discuss in this paper combines both approaches in a novel manner; moreover, it takes advantage of the outstanding progresses in high-rate radiation-hard detectors that have been achieved for the calorimetry in the forward region of LHC experiments and for the study of very rare kaon decays. As in [8], the tagging setup discussed hereafter is especially suited to suppress the $v_{e}$ contamination in $v_{\mu}$ beams from the decay in flight of multi-GeV pions, i.e. to reduce the intrinsic contamination of beam-related $v_{e}$ events at experiments seeking for $v_{\mu} \rightarrow v_{e}$ transitions. It can also be employed to reduce the systematic error in the knowledge of the flavor contamination at source or to select a pure $v_{e}$ subsample for physics studies. The physics reach of such experiments ranges from the study of anomalous short baseline oscillations [9-13] and low energy cross sections [14], which employs low power proton beams on solid targets, up to ambitious "Superbeam" facilities [15-20] utilizing MWclass proton drivers to address the study of subdominant $v_{\mu} \rightarrow v_{e}$ transitions at the atmospheric scale. All these experiments are limited by systematic errors [21, 22] mainly arising from the finite purity of the neutrino source: as a consequence, in the last decade the development of novel facilities designated to overcome the purity constraints of $\pi$-based beams has been at focus of intense R\&D efforts. ${ }^{1}$ In this paper, the tagging principle and the conceptual design of a facility aimed at a substantial reduction of the $v_{e}$ contamination is discussed in Sect. 2. Simulation and performance for background rejection in a setup "scraping" the secondary beam until the hadron dump is studied in Sect. 3.

${ }^{1}$ For a detailed review, see the Reports of the "International Scoping Study of a future Neutrino Factory and Super-beam facility" [23-25]. 
Finally, the special case of an additional instrumented beam dump at the end of the decay tunnel is discussed in Sect. 4.

\section{Tagging of electron neutrinos}

Artificial sources of $v_{\mu}$ at energies larger than $\sim 100 \mathrm{MeV}$ can be produced by the two-body decay in flight of pions $\pi^{+} \rightarrow \mu^{+} v_{\mu}$, which in turn are created from protons impinging on thick targets [26]. The source also contains $v_{\mu}$ originating from two-body and three-body decays of charged and neutral kaons. However, it is intrinsically polluted by $v_{e}$ originating from three-body decays of $K^{+}$ $\left(K_{e 3}: K^{+} \rightarrow \pi^{0} v_{e} e^{+}\right)$, from the decay-in-flight of secondary muons along decay tunnel (DIF: $\pi^{+} \rightarrow \mu^{+} v_{\mu} \rightarrow$ $\left.e^{+} v_{e} v_{\mu} \bar{v}_{\mu}\right)$ and from the decays of neutral kaons. Tuning of the pion momentum selected by magnetic lenses after the primary target, of the transfer line up to the decay tunnel and, finally, of the length and radius of the tunnel itself help in reducing the ratio $v_{e} / v_{\mu}$ below $1 \%$, although a $v_{e}$ contaminations in the $1-0.1 \%$ range is unavoidable in any realistic configuration. In a very broad neutrino energy range, i.e. from sub-GeV [27] up to tens of $\mathrm{GeV}$ [28], the $v_{e}$ contamination is dominated by $\pi^{+}$DIF and by $K_{e 3}$, with the addition of a minor contribution from semileptonic decay of $K_{L}^{0}\left(K_{L}^{0} \rightarrow \pi^{-} e^{+} \nu_{e}\right)$ and charged kaons decaying before the bending magnets. All these decays produce positrons in the final state, whose spectral distribution follows from three-body kinematics and from the specific Qvalue of the reaction. In the forward region along the decay tunnel, positrons are swamped by the bulk of undecayed hadrons, by muons resulting from the two-body decay of $\pi^{+}$ and by secondary protons within the acceptance of the focusing system. Figure 1 shows the polar angle distribution $\theta$ of positrons, muons and electrons with respect to the axis of the decay tunnel for a specific beam configuration ("benchmark beamline"-see Sect. 3) resulting from the decay in flight of $\pi^{+}$and $K^{+}$with $8.5 \mathrm{GeV}$ mean energy. At the entrance of the decay tunnel, mesons and protons are assumed to be uniformly distributed in a $10 \times 10 \mathrm{~cm}^{2}$ area with a polar angle smaller than 3 mrad (black solid curve in Fig. 1left). On top of the beam divergence, positrons from $K_{e 3}$ (Fig. 1-right) show a large intrinsic divergence due to threebody kinematics, with a mean $\theta$ value of $88 \mathrm{mrad}$. The mean polar angle of muon-neutrinos produced by the source is of the order of $27 \mathrm{mrad}$ (blue dot-dashed line in Fig. 1-left), i.e. it has a value intermediate between the $\pi^{+}$and positron beam divergence. Similarly, the $\theta$ distribution of positrons from DIF has a mean value of $28 \mathrm{mrad}$ (black solid line of Fig. 1-right).

On the other hand, the polar angle of the accompany muons in $\pi^{+} \rightarrow \mu^{+} v_{\mu}$ along the decay tunnel is extremely small (red dashed in Fig. 1-left) and, in fact, comparable with the beam divergence of the parent $\pi^{+}$. This muon focusing effect is due to 2-body kinematics and to the fact that the rest mass of the muon is comparable with the pion rest mass. The emission angle $\theta$ of the muon is, therefore,

$\tan \theta=\frac{\sin \theta^{*}}{\gamma\left(1 / \beta^{*}+\cos \theta^{*}\right)}$

$\gamma$ being the Lorentz boost of the parent pion $(\beta \simeq 1)$ in the laboratory frame, while $\theta^{*}$ and $\beta^{*}=\left(m_{\pi}^{2}-m_{\mu}^{2}\right) /\left(m_{\pi}^{2}+\right.$ $m_{\mu}^{2}$ ) $\simeq 0.26$ are the emission angle and the muon velocity in the pion rest frame, respectively. On the contrary, $\beta^{*}=1$ for neutrinos; hence, the emission angle in the laboratory frame at large $\theta^{*}\left(\cos \theta^{*} \simeq 0\right)$ is much wider than for $\mu^{+}$.
Fig. 1 (Color online) Left: $\theta$ angle distribution between the propagation direction of pions (black solid line), muons (red dashed) and $v_{\mu}$ (blue dot-dashed) and the axis of the decay tunnel. Right: $\theta$ distribution for positrons resulting from DIF (black solid line) and $K_{e 3}$ decays (red dashed line)

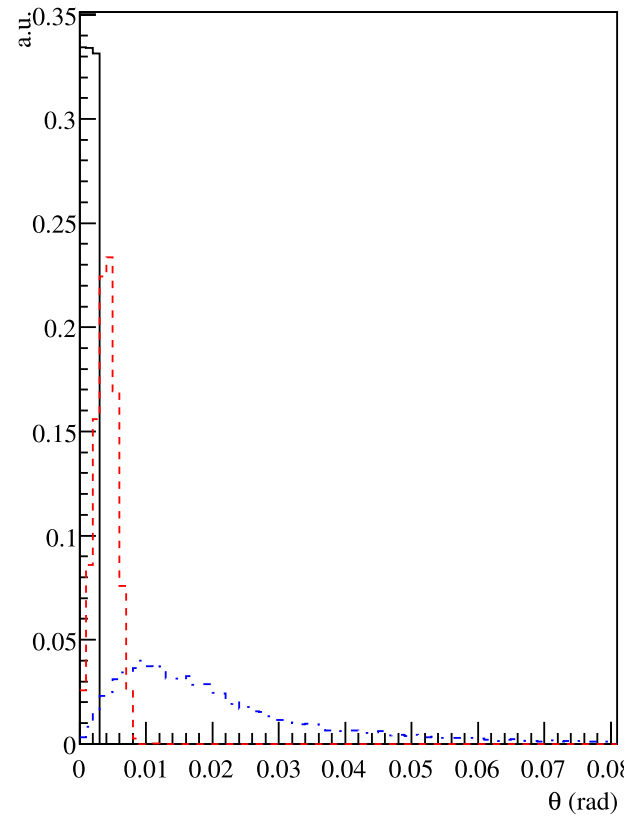


The above considerations point toward a tagging setup designed to perform a destructive (calorimetric) measurement of the positrons and intercepting secondary particles emerging from the primary pion beam up to the end of the decay tunnel. The setup is shown schematically in Fig. 2. It consists of a set of cylindrical e.m. calorimeters with a geometry and readout similar to the ATLAS forward calorimeter (FCAL [30]) but with much shorter length $\left(10 X_{0}\right)$. The FCAL is a liquid Argon calorimeter designed to operate in a high radiation density environment. It ensures radiation hardness up to $0.5 \mathrm{GRad} / \mathrm{y}$, fast response to cope with the $25 \mathrm{~ns}$ beam crossing of the LHC and reduced sensitivity to event pile-up thanks to a very small drift length $(0.27 \mathrm{~mm})$. Such small gap allows for full particle drift in $61 \mathrm{~ns}$ and, therefore, relieves the detector of the problem of ion buildup [29]. Unlike ATLAS, the modules considered here are built with inner radii of variable size, so that all primary mesons and most secondary muons reach the beam dump without intercepting the calorimeters. Differently from early proposals $[3,8]$ there is no material installed along the trajectory of the undecayed pions up to the end of the decay tunnel; therefore, irrespective to the tagging performance, the flux of $v_{\mu}$ at the far detector remains unchanged after the installation of the modules. In front of each module, a high speed tracker of granularity comparable to the FCAL $(\sim 1 \mathrm{~cm})$ is envisaged. The tracker provides an absolute time-stamp of the incoming candidate positron with a required precision of $\sim 1 \mathrm{~ns}$ (see Sect. 3) and vetoes neutral energy deposits due to energetic photons from $\pi^{0}$ or hard bremsstrahlung. In the occurrence of a neutrino interaction at the far location, a time coincidence with a charged particle in the tracker is sought for, once accounting for the propagation delay due to the source to the detector distance. In
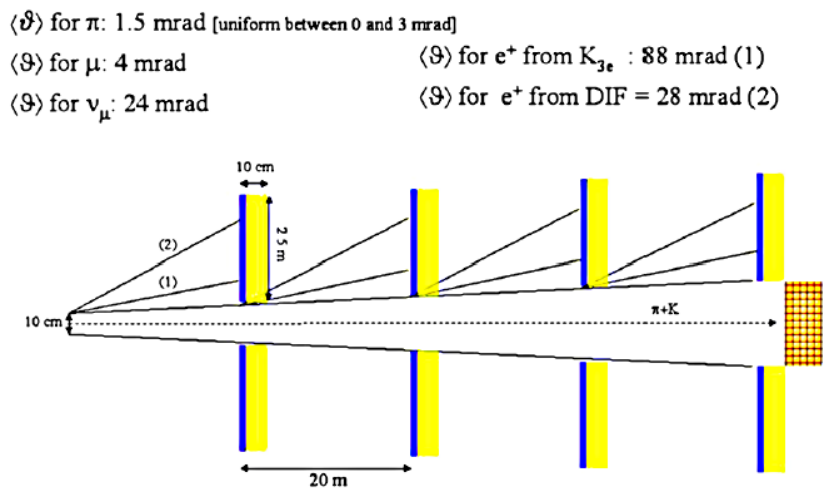

Decay tunnel: $80 \mathrm{~m}$

Fig. 2 (Color online) Schematics of the tagging setup (lateral view, not in scale). The blue\&yellow (solid) boxes indicate the position and size of the tagging modules (tracker and calorimeter) along the $80 \mathrm{~m}$ decay tunnel. The lines labeled (1) and (2) show the average $\theta$ angles for positrons from $K_{e 3}$ and DIF, respectively. The orange (gridded) box represents the instrumented dump (Sect. 4) addition, an electromagnetic deposit beyond a given threshold is required in the FCAL area adjacent to the tracker hits within a time window comparable with the drift time of the calorimeter. The presence of such energy deposit indicates the simultaneous production of a neutrino with a positron, tagging the event at the far location as a " $v_{e}$ at source". The finite efficiency and geometrical acceptance of the modules (e.g. due to positron produced along the pion flight direction) limits the capability to veto $v_{e}$ (Sect. 3.1). Fake vetoes due to accidental overlaps of muons with photons, e.m. shower leakage along the modules and $\mu \rightarrow e$ misidentification induces additional dead-time, causing a drop in statistics (Sect. 3.2). As discussed in Sects. 3 and 4, in order to achieve a $v_{e}$ suppression rate of about one order of magnitude and a sizable detector livetime, the tracker must be able to operate with rates up to $\sim 200 \mathrm{kHz} / \mathrm{cm}^{2}\left(\sim 20 \mathrm{MHz} / \mathrm{cm}^{2}\right.$ in the proximity of the beam dump) and a time resolution better than $1 \mathrm{~ns}$. Such requirements point toward the use of fast semiconductor detectors ${ }^{2}$ : they share with collider physics applications the constraints coming from radiation hardness [31] and with rare kaon decay physics applications the need for small material budget and sub-ns time resolution [32]. However, the granularity requirements are highly reduced $(1 \mathrm{~cm}$ versus a few hundreds $\mu \mathrm{m})$ and the constraints on the time resolution can be relaxed by about one order of magnitude ( $1 \mathrm{~ns}$ versus $100 \mathrm{ps}$ ); still, fast trackers for neutrino tagging applications represent a technological challenge due to the large area needed for full coverage of the calorimeters in the region where particle rates are high $\left(\sim 10 \mathrm{~m}^{2}\right.$ for rates higher than $\left.10 \mathrm{kHz} / \mathrm{cm}^{2}\right)$. The requirement of a time resolution better than $1 \mathrm{~ns}$ poses strict constraints to the neutrino detector, as well, thus narrowing the choice of technologies that can be employed. Cherenkov detectors, in particular, offer the advantage of extremely fast response at the expense of reduced light yield with respect to scintillators or gaseous detector. In recent years, water Cherenkov neutrino detectors have achieved resolutions below $1 \mathrm{~ns}$ for masses up to 50 kton [33], although fast triggering from liquid and solid scintillators, from UV light in liquefied noble gases and from gaseous detectors still represent viable alternatives. Finally, it is worth noticing that an absolute time calibration between the instrumented decay tunnel and the neutrino detector must match the abovementioned resolutions. For short baseline experiments, it can easily be achieved with atomic clocks, while the technique currently exploited by long baseline experiments and based on the Global Positioning System [34-36] does not fulfill this constraint ( $\Delta t \simeq 10 \mathrm{~ns}$ ), so that a direct resynchronization of the clocks would be periodically needed.

\footnotetext{
${ }^{2}$ Other options based on scintillators or gaseous detectors can be envisaged, especially in the areas far from the secondary meson beam, where the requirements on the particle rate can be relaxed below $10 \mathrm{kHz} / \mathrm{cm}^{2}$ (see Sect. 3).
} 


\section{Performance of the tagging system}

\subsection{Event selection and tagging efficiency}

The tagging concept outlined in Sect. 2 has been tested against a specific beam configuration in order to quantify the performance of the $v_{e}$ suppression. As already mentioned, the two most relevant contributions to the $v_{e}$ beam contamination are due to $K_{e 3}$ and DIF. The beam setup considered hereafter ("benchmark beamline") is similar to the one studied in [8] and it is suited for short baseline $v_{e}$ appearance searches, along the line of [9-13]. In fact, the beamline of [8] has been considered as an upgrade of the I216 Proposal [37] at the CERN PS; here, it is used as a benchmark and the number of pions produced per extraction, together with the proton mean power are considered as free parameters. The neutrino beam in this benchmark configuration is produced by a slow extraction of protons ( $1 \mathrm{~s})$ from a $19.2 \mathrm{GeV}$ booster. In our simulation [41] protons are dumped in a cylindrical beryllium target $(110 \mathrm{~cm}$ length and $3 \mathrm{~mm}$ diameter) producing secondary particles, which are momentum-selected and transported up to the decay tunnel by a magnetic focusing system. The focusing system necessarily relies on quadrupole-dipole magnets [26] due to the long extraction spill and has not been simulated in details (halo muons and off-momentum particles are, therefore, neglected). Similarly to [8], we assume the focusing system to have an angular acceptance of $80 \mu \mathrm{Sr}$ and a momentum bite of $\pm 20 \%$ around a nominal momentum of $8.5 \mathrm{GeV}$. All selected secondary particles are focused at the entrance of the decay tunnel, uniformly distributed in a $10 \times 10 \mathrm{~cm}^{2}$ window. Here, in order to simulate the beam divergence, the secondary particles are randomly distributed up to an angle of $3 \mathrm{mrad}$ with respect to the axis of the decay tunnel. We cross-checked our simulation with a Sanford-Wang [38] parametrization of pion production data [39] and with $\pi^{+}$ and $K^{+}$data taken with $19.2 \mathrm{GeV}$ proton on beryllium [40]. The decay tunnel is $80 \mathrm{~m}$ long with a $2.5 \mathrm{~m}$ radius (Fig. 2). We neglect here the production of $K_{L}^{0}$ and $K^{-}$at the target, whose $v_{e}$ contribution at the far detector depends on the details of the focusing system. With respect to traditional neutrino beams, this contribution is further suppressed due to the bending between the primary target and the decay tunnel; it is, thus, negligible with respect to the $K_{e 3}$ and DIF contributions even after tagging suppression. Assuming a primary beam intensity of $2 \times 10^{13}$ protons-on-target (pot) per extraction [8], we evaluate from simulation a particle density at the entrance of the decay tunnel of about $R_{0} \equiv 100 \mathrm{MHz} / \mathrm{cm}^{2}$ during the $1 \mathrm{~s}$-long spill. It corresponds to $N_{\pi}=10^{10} \pi^{+}$per extraction over the whole surface, with a mean $K^{+} / \pi^{+}$of $4.1 \%$. Any increase of the neutrino flux achieved by an increase of the time the booster is dedicated to the neutrino beamline ("duty cycle") does not affect the tagging performance. ${ }^{3}$ However, a power increase obtained raising the number of mesons $R_{0}$ per extraction, e.g. increasing the proton beam intensity and energy or the acceptance of the focusing system, increases the particle rate at the taggers and challenge the tagging performance. For the benchmark beamline in our simulation, Fig. 3 shows the spectra of all $\pi^{+}$and $K^{+}$produced and crossing a circle of $1.4 \mathrm{~m}$ radius, located $2 \mathrm{~m}$ downstream the target and the spectra of $\pi^{+}$and $K^{+}$accepted by the focusing and bending system. The neutrino flux in a far detector located $800 \mathrm{~m}$ from the decay tunnel is also shown in Fig. 3. The neutrino beam is a narrow-band beam of $1.25 \times 10^{-7} \mathrm{v} / \mathrm{pot} / \mathrm{m}^{2}$ at a far detector. It gives $\sim 1.9 \times 10^{4}$ events every $10^{20}$ pot in a 1 kton detector. The mean $v_{\mu}$ energy is $3.5 \mathrm{GeV}$ and the $v_{e}$ contamination is $0.1 \%$.

Along the decay tunnel, four tagging stations have been simulated. They are located $z=20,40,60$ and $80 \mathrm{~m}$ far from the entrance of the tunnel $(z=0)$. The stations have a cylindrical geometry with $2.5 \mathrm{~m}$ outer radius and variable inner radii of 12, 17, 21 and $26 \mathrm{~cm}$, corresponding to an angular opening of $6,4.25,3.5$ and 3.25 mrad. Variable angular openings are employed since the source at $z=0$ is non-pointlike: in this case most of undecayed primary $\pi^{+}$ and $K^{+}$reach the beam dump at $z=80 \mathrm{~m}$ through the central holes of the modules and only secondaries are scraped

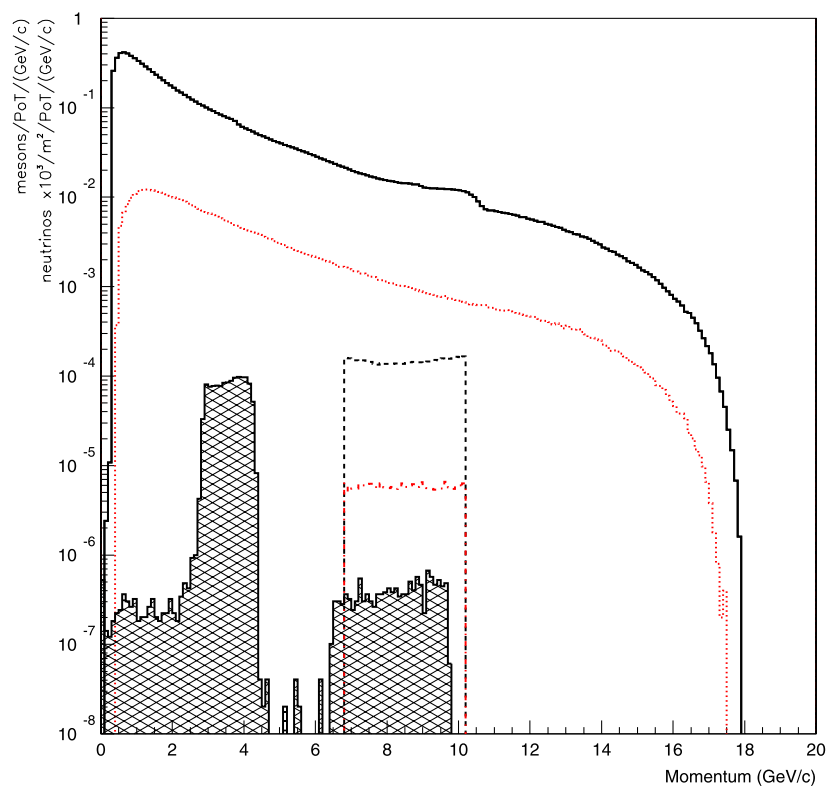

Fig. 3 (Color online) Muon neutrino flux (hatched histogram) in a detector located $800 \mathrm{~m}$ from the target. Spectra of $\pi^{+}$(black solid line) and $\mathrm{K}^{+}$(red dotted) $2 \mathrm{~m}$ downstream the target. Spectra of $\pi^{+}$(black dashed) and $K^{+}$(red dot-dashed) selected by the focusing and bending system

${ }^{3}$ For the benchmark beamline $\left(E_{p}=19.2 \mathrm{GeV}\right)$, a $1 \mathrm{~s}$ long extraction delivering $2 \times 10^{13}$ pot every $7 \mathrm{~s}$ corresponds to a mean beam power of $9 \mathrm{~kW}$ and a duty cycle of $1 / 7=14 \%$. 
by the tagging units. Each module has a material budget of $10 X_{0}$ of lead, ${ }^{4}$ which has been simulated in GEANT4 $[42,43]$. The calorimeter response to energy deposit has not been simulated in details: for e.m. deposits (positrons, electrons, photons) the reconstructed energy is drawn from the deposited energy and smeared according to the FCAL measured resolution [44]. In the present case, the energy resolution is dominated by the sampling term, which is assumed to be $30 \% / \sqrt{E}$ with $E$ expressed in $\mathrm{GeV}$. Since the tagger thickness is just $33 \%$ of the $\mathrm{Pb}$ interaction length, pions deposit only a small fraction of their energy in the tagger. Such deposit is mip-like in about $74 \%$ of the case and it exhibits strong energy fluctuations in the occurrence of hard hadronic interactions. It is shown in Fig. 4-left (black line) and superimposed with the energy deposit of muons from $\pi^{+}$decay (red dashed line).

Figure 4-right shows the reconstructed energy of positrons from $K_{e 3}$ and DIF, when a $v_{e}$ reaches the far detector. The energy has been smeared according to the FCAL resolution and accounting for lateral leakage. For each event we require a $v_{e}$ with energy larger than $0.5 \mathrm{GeV}$ hitting the far detector within its geometrical acceptance. We assumed a sourceto-detector distance of $800 \mathrm{~m} \mathrm{[8].} \mathrm{The} \mathrm{detector} \mathrm{surface} \mathrm{in}$ the plane perpendicular to the neutrino beam is $10 \times 10 \mathrm{~m}^{2}$. Lateral leakage is marginal $(\sim 1 \%)$ for positrons if all energy deposited within a $2 \mathrm{~cm}$ radius $\left(R_{c}\right)$ around the impact point is collected. $R_{c}$ has been optimized empirically: larger collecting radii are detrimental for $\pi / e$ separation and for the effect of event pile-up while radii much smaller than $2 \mathrm{~cm}$ reduce the visible energy of the positrons, which gets closer to a mip-like deposit.

Finally, a " $v_{e}$ at source" is defined as a charged particle triggered by the tracker with an energy deposit in the calorimeter greater than $300 \mathrm{MeV}$, in coincidence with a $v_{e}$ interactions at the far detector. Again, only neutrinos with energy larger than $0.5 \mathrm{GeV}$ are considered. The inefficiency of the tracker has been neglected together with subdominant effects as the albedo resulting from backscattered electrons or the different energy response for the hadronic and e.m. component in the core $\left(R_{c}<2 \mathrm{~cm}\right)$ of the $\pi^{+}$shower. Results in terms of $K_{e 3}$ and DIF suppression are listed in Table 1 . The numbers in bold indicate the veto efficiency for events giving a $v_{e}$ at the far detector coming from $K_{e 3}$ and DIF, respectively. The tagging system, therefore, is powerful in vetoing the $K_{e 3}$ contamination, while the performance are poorer for DIF $v_{e}$. This is due both to the smaller angular spread of DIF $e^{+}$and to the different lifetime of kaons and muons. Since the $\gamma c \tau$ of the kaon $(64 \mathrm{~m})$ is comparable with the length of the decay tunnel, $v_{e}$ from $K_{e 3}$ decay are produced earlier than $v_{e}$ from DIF, generating positrons that are

\footnotetext{
${ }^{4}$ In fact, in the special case of the Atlas FCAL, copper has been employed as passive material.
}

intercepted by the tagging modules. On the other hand, due to the longer pion and muon lifetime $\left(\gamma_{\pi} c \tau_{\pi} \simeq 478 \mathrm{~m}\right)$ most DIF occur in the proximity of the beam dump and positrons impinge upon the dump at a radius smaller than the inner radius of the last tagging unit. In Table 1, the improvement in vetoing the DIF is shown in the third column, assuming that the last tagging unit has no inner hole, i.e. instrumenting the beam dump (see Sect. 4), and applying a tighter cut of $1 \mathrm{GeV}$ in this area. As anticipated, the improvement in the $K_{e 3}$ rejection is marginal $(+3 \%)$ while the rejection of DIF increases substantially $(+16 \%)$. The second and fourth columns are computed without energy smearing ("NS") and show the impact of the sampling term of the e.m. calorimeter on the tagging performance. Finally, it is worth noticing that, beyond tagging, the instrumented decay tunnel allows for a precise measurement of the secondary beam and, therefore, it contributes to reduce systematic errors on the $v$ flux and composition.

\subsection{Particle rate and accidental coincidences}

The particle rate at the tagging units is clearly dominated by the 2-body decay of the $\pi^{+}$with $\mu^{+}$impinging on the calorimeters along the decay tunnel. In the beam dump (see Sect. 4), it mainly depends on the rate of undecayed $\pi^{+}$, plus a small correction due to $K^{+}$and secondary protons that reaches the end of the beamline. Figure 5 shows the average particle rate during the $1 \mathrm{~s}$ extraction as a function of the tagger radius. As anticipated, the rate at the taggers is dominated by muons, with peak values of about $200 \mathrm{kHz} / \mathrm{cm}^{2}$. As a consequence, the peak rate of pile-up is

$P R=2 \times 10^{5} \mathrm{~cm}^{-2} \mathrm{~s}^{-1} S \Delta T_{\text {cal }}$

$S$ being the collection surface $\pi R_{c}^{2} \simeq 12 \mathrm{~cm}^{2}$ and $\Delta T_{\text {cal }}$ is the integration time of the detector. For the above-mentioned FCAL, it corresponds to $61 \mathrm{~ns}$ if operated in full-drift mode, but it drops below $25 \mathrm{~ns}$ in the standard LHC readout configuration, which exploits the fast rise of the signal. Since piling-up particles are mainly constituted by muons, PR up to $\sim 3$ are sustainable before the energy deposit reaches the threshold for positron identification $(300 \mathrm{MeV})$. For the benchmark beamline $P R \simeq 0.06$, so that beam powers up to a few hundreds of $\mathrm{kW}$ would be sustainable. In particular, a pile-up of 3 is reached by a beam power of $330 \mathrm{~kW}$, assuming a duty cycle similar to the one of the benchmark beamline (14\%).

Accidental coincidences are due to events classified as " $v_{e}$ at source" by the tagging system in the same time window ( $\sim 1 \mathrm{~ns})$ of the neutrino interaction at the far location. Two classes of events give contributions.

Firstly, accidental tags can arise from genuine $v_{e}$ at source in coincidence with $v_{\mu}$ or $v_{\mu} \rightarrow v_{e}$ interactions in the detector. The probability of having a fake coincidence 
Fig. 4 (Color online) Left: energy at the tagger for undecayed $\pi^{+}$(black solid line) and $\mu^{+}$(red dashed). Right: energy at the tagger from $e^{+}$ originating by $K_{e 3}$ (red dashed) and DIF (black solid) giving a $v_{e}$ at the far detector (" $v_{e}$ at source")

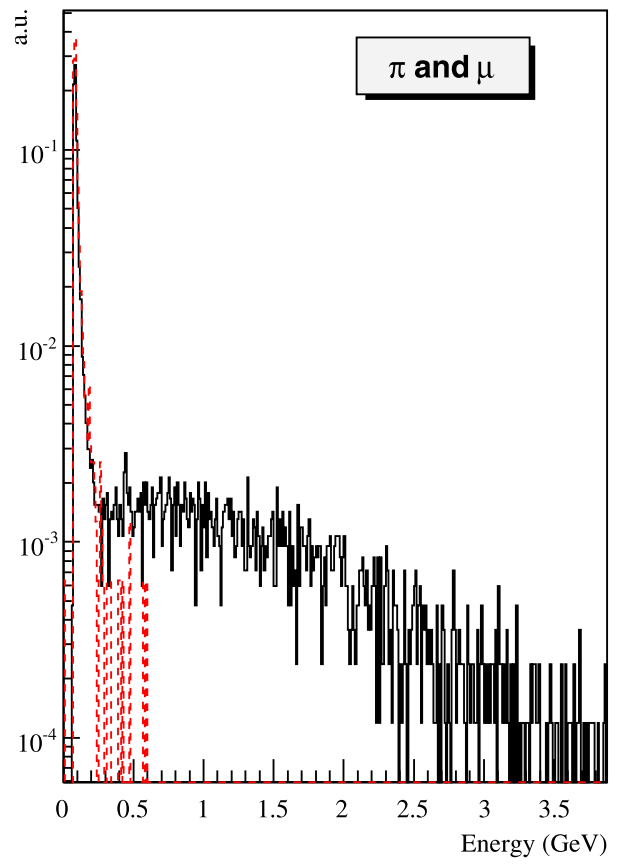

Table 1 Percentage of $v_{e}$ from $K_{e 3}$ (first line) and DIF (second line) at source vetoed by the tagging system. The first two columns indicate the tagging efficiency assuming only the scraping of the secondary beam as described in the text. NS (no-smearing) shows the efficiencies obtained considering an ideal e.m. calorimeter (i.e. a negligible sampling term). The last two columns show the improvement gained introducing an instrumented beam-dump and applying a tighter cut of $1 \mathrm{GeV}$ on the reconstructed energy inside the dump region $(R<26 \mathrm{~cm}$ at the last tagging module). Errors are due to finite MC statistics

\begin{tabular}{lllll}
\hline & Scraping & Scraping (NS) & Dump & Dump (NS) \\
\hline$K_{e 3}$ & $\mathbf{8 6 . 2} \pm \mathbf{0 . 3} \%$ & $88.5 \pm 0.3 \%$ & $89.1 \pm 0.3 \%$ & $91.4 \pm 0.3 \%$ \\
DIF & $\mathbf{6 0 . 7} \pm \mathbf{0 . 7} \%$ & $64.4 \pm 0.7 \%$ & $76.1 \pm 0.6 \%$ & $80.0 \pm 0.6 \%$ \\
\hline
\end{tabular}

between an event at the far detector and a " $v_{e}$ at source" from the tagging system is given by the rate of positrons impinging on the trackers times the squared sum of the time resolution of the tracker and the neutrino detector $(\Delta t)$. The rate of accidentals is, therefore,

$\left[N_{\pi} f_{e^{+}}^{\pi} \epsilon_{\mathrm{DIF}}+N_{K} f_{e^{+}}^{K} \epsilon_{K_{e 3}}\right] \cdot \Delta t \simeq 1.3 \times 10^{7} \cdot \Delta t ;$

$\epsilon_{\mathrm{DIF}}$ and $\epsilon_{K_{e 3}}$ are the tagging efficiency for DIF and $K_{e 3}$ positrons, ${ }^{5} N_{\pi} \simeq 10^{10} \mathrm{~s}^{-1}$ the rate of pions at $z=0$ and $N_{K} \simeq 4.1 \times 10^{-2} \cdot N_{\pi}$ the kaons at $z=0$. The fraction of $\pi^{+}\left(K^{+}\right)$giving a positron in the decay tunnel from DIF $\left(K_{e 3}\right)$ is labeled $f_{e^{+}}^{\pi}\left(f_{e^{+}}^{K}\right)$ and, in the benchmark beamline is $\simeq 0.017 \%$ (3.4\%). In particular, $f_{e^{+}}^{K}=B R\left(K_{e 3}\right)(1-$ $\left.e^{-\gamma_{K} c \tau_{K} / L}\right), L$ being the tunnel length. Equation (3) sets the

\footnotetext{
${ }^{5}$ Unlike Table 1 , these efficiencies (63\% and $83 \%$ respectively) are computed for all positrons, irrespective of the energy and direction of the outcoming $v_{e}$.
}

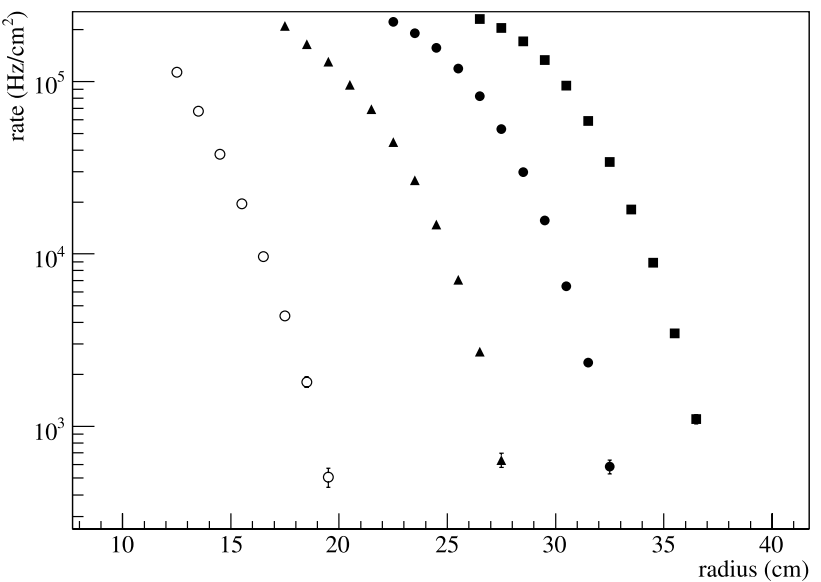

Fig. 5 Rate at the first (20 m distance-empty dots), second (40 m, triangles $)$, third $(60 \mathrm{~m}$, full dots) and fourth $(80 \mathrm{~m}$, squares $)$ tagging module. The error bars (visible only at large radii) show the errors due to finite MC statistics

scale of the time resolution needed at the tracker and neutrino detector. For $\Delta t=1 \mathrm{~ns}$, the fraction of $v_{\mu} \rightarrow v_{e}$ interactions at the far detector wrongly tagged as " $\nu_{e}$ at source" is $1.3 \%$, i.e. the effective livetime of the detector is about $99 \%$. It sets more stringent limit than pile-up to the scalability of this technique up to the Superbeams: already an order of magnitude increase of $N_{\pi}(100 \mathrm{~kW}$ for a $14 \%$ duty cycle) would bring the livetime below $70 \%$. To cope with Superbeam powers, time resolutions of the order of a few hundreds of ps would be needed both at the tracker and at the neutrino detector.

Finally, it is worth noting that (3) demonstrates quantitatively what stated in Sect. 2, i.e. the need of a high speed 
Table 2 Sources of background and their contribution to the probability of false tagging

\begin{tabular}{lllc}
\hline Source & BR & Misid & Fake tag prob \\
\hline$\pi^{+} \rightarrow \mu^{+} v_{\mu}$ & $100 \%$ & $\mu \rightarrow e$ misid. & $0.3 \%$ \\
$\mu^{+} \rightarrow e^{+} \bar{v}_{\mu} v_{\mu}$ & DIF & genuine $e^{+}$ & $0.1 \%$ \\
$K^{+} \rightarrow \mu^{+} v_{\mu}$ & $63.5 \%$ & $\mu \rightarrow e$ misid. & $<0.1 \%$ \\
$K^{+} \rightarrow \pi^{+} \pi^{0}$ & $20.7 \%$ & $\pi \rightarrow e$ misid. & $1.4 \%$ \\
$K^{+} \rightarrow \pi^{+} \pi^{+} \pi^{-}$ & $5.6 \%$ & $\pi \rightarrow e$ misid. & $0.7 \%$ \\
$K^{+} \rightarrow \pi^{0} e^{+} v_{e}$ & $5.1 \%$ & genuine $e^{+}$ & $1.1 \%$ \\
$K^{+} \rightarrow \pi^{0} \mu^{+} v_{\mu}$ & $3.3 \%$ & $\mu \rightarrow e$ misid. & $<0.1 \%$ \\
$K^{+} \rightarrow \pi^{+} \pi^{0} \pi^{0}$ & $1.7 \%$ & $\pi \rightarrow e$ misid. & $<0.1 \%$ \\
\hline
\end{tabular}

tracker in front of the calorimeter units with time resolution of $\sim 1 \mathrm{~ns}$. Relying on the calorimetric measurement only would increase the rate of accidental coincidences by about one order of magnitude.

The second source of accidentals comes from events without positrons in the final state misidentified as " $v_{e}$ at source" due to finite detector resolution $(\mu \rightarrow e$ or $\pi \rightarrow e$ misidentification). For the bulk of muons in the 2-body decay of $\pi^{+}$, the misidentification probability is $5.4 \times 10^{-3}$, resulting into a probability of a fake veto of $0.3 \%$. However, two-body hadronic decays of $K^{+}$can give a relatively large rate of false tagging. This is due to the fact that pions are produced at large angles in association with photons from $\pi^{0}$ decay. Since the $\pi \rightarrow e$ misidentification probability is large $(\sim 25 \%$, see Fig. 4$)$ the accidental rate of $K^{+} \rightarrow \pi^{+} \pi^{0}(1.4 \%)$ is comparable with the rate due to genuine $v_{e}$ sources. Clearly, the poor $\pi / e$ rejection capability in the tagger is due to the fact that the longitudinal development of the hadronic versus e.m. shower is not exploited for $\pi / e$ separation (see Sect. 4). Other sources of background are listed in Table 2 and do not exceed $1 \%$.

\section{Performance of an instrumented beam dump}

As shown in Sect. 3, the performance of the tagging system are excellent for the suppression of the $v_{e}$ from $K_{e 3}$ while a significant fraction of the positrons produced by the decay-in-flight of muons impinges upon the hadron dump at the end of the decay tunnel with a radius smaller than the inner radius of the last tagging station. Figure 6 shows the particle rate at the last tagging module at radii smaller than $R_{\min }=26 \mathrm{~cm}$. For the benchmark beamline, the rates do not exceed $20 \mathrm{MHz} / \mathrm{cm}^{2}$, so that it would be conceivable to instrument also the dump with a fast tracker followed by a FCAL unit. Three issues, however, have to be addressed, which in principle limit the tagging performance of the dump and its scalability to higher powers. The first is related to the integrated dose. Unlike the scraping modules, the dump is crossed by the bulk of undecayed pions

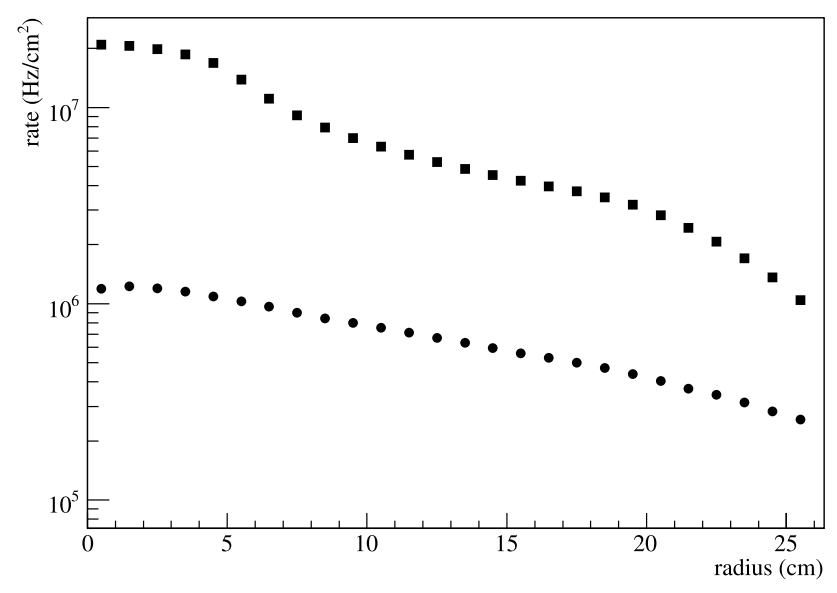

Fig. 6 Charged particle rate (squares) and muon rate (dots) at the last module for $R<26 \mathrm{~cm}$ (instrumented beam dump)

and by secondary protons that deposit an average energy fraction $\epsilon_{\pi}$ and $\epsilon_{p}$ of their initial energy. The value of $\epsilon_{\pi, p}$ has been computed by simulation and it turns out to be $\epsilon_{\pi}=5.2 \pm 0.5 \%$ for pions and $\epsilon_{p}=4.0 \pm 0.5 \%$ for protons in $10 X_{0}$ of $\mathrm{Pb}$. In the benchmark beamline the proton yield at the dump is large and comparable with the one of the undecayed pions: for each extraction $1.5 \times 10^{10}$ protons are distributed at the entrance of the decay tunnel in the $10 \times 10 \mathrm{~cm}^{2}$ surface. On the other hand, if the beamline is operated in antineutrino mode $\left(\pi^{-}\right.$selection after the primary target), the corresponding antiproton contribution is below the one of $K^{-}$. Neglecting the small contribution of undecayed $K^{+}$, the yearly integrated dose is, therefore

$$
\begin{aligned}
& \frac{N_{\pi}\left[(1-f) \epsilon_{\pi} E_{\pi}+f E_{\mathrm{mip}}\right]+N_{p} \epsilon_{p} E_{p}}{M} \\
& \simeq 1.3 \times 10^{-2} \mathrm{~Gy} / \mathrm{spill}
\end{aligned}
$$

which corresponds to $25 \mathrm{kGy}$ for a module weight of $M=$ $115 \mathrm{~kg}\left(10 X_{0}\right.$ of $\left.\mathrm{Pb}\right)$ and $10^{20}$ pot. In (4) $f$ represents the fraction of decayed pions (15\%), $E_{\pi}$ and $E_{p}$ the mean pion and proton energy and $E_{\text {mip }}$ the energy released by a muon in $10 X_{0}$. The integrated dose is, therefore, much below the safety operation limit of FCAL (5 MGy/y [30]).

Once more, the issue that poses the main impediment to installing an instrumented dump at higher beam powers is the rate of false tags from random coincidences. Here, the number of accidentals at the far detector per neutrino interaction scales as:

$\left[N_{\pi}(1-f) \epsilon_{\pi \rightarrow e}+N_{p} \epsilon_{p \rightarrow e}\right] \cdot \Delta t \simeq 23 \cdot \epsilon_{\pi \rightarrow e}$

with $\Delta t \simeq 1 \mathrm{~ns}$ and $\epsilon_{\pi \rightarrow e}\left(\epsilon_{p \rightarrow e}\right)$ representing the fraction of pions (protons) identified as electrons. In this case, even at low power (benchmark beamline), the $\pi / e$ misidentification rate must be kept well below the value measured at the tagger (see Sect. 3.2). This is quite an ambitious task, since the 
energy of the pions is much larger than the positron spectrum in DIF events and, in order to reduce pile-up, only a small area around the impact point can be used to collect the deposited energy. In this case, better performance can be obtained aligning two FCAL modules of different thickness: the first one (FCAL1) is a standard $10 X_{0}$ tagger module, which is followed by a second hadronic module FCAL2 of about three interaction lengths (thickness: $51 \mathrm{~cm}$ ). This configuration is quite similar to the one employed in the ATLAS forward region [30, 45]. In this case, a more powerful $\pi / e$ and $p / e$ separation can be achieved selecting events in the $E 1$ versus $E 2$ plane (see Fig. 7), $E 1$ and $E 2$ being the visible energy deposited at FCAL1 and FCAL2, respectively. Again, the energy resolution for positrons is dominated by the sampling term of the calorimeter while the hadronic resolution is dominated by the lateral leakage of the hadronic energy at $R_{c}>2 \mathrm{~cm} . \epsilon_{\pi \rightarrow e}$ has been computed from the distribution of $E 1$ and $E 2$ for events selected with the cut of Table 1, third column, ("Dump"), i.e. requiring a visible energy deposit in FCAL1 greater than $1 \mathrm{GeV}$ and a small deposit $(<300 \mathrm{MeV})$ in FCAL2. Here, the misidentification probability $\epsilon_{\pi \rightarrow e}$ drops to $3 \%$. This value is still too high for the benchmark beamline, especially when neutrinos are produced and the proton contamination has to be accounted for (see (5)).

Finally, the tagging performance of the dump is challenged by event pile-up, which is in fact entangled with the evaluation of $\epsilon_{\pi \rightarrow e}$. For the benchmark beamline, even neglecting the proton contribution, the pion density at the dump is $N_{\pi}(1-f) / \pi R_{\min }^{2} \simeq 4 \mathrm{MHz} / \mathrm{cm}^{2}$. So, for an effective integration window $\Delta T_{\text {cal }}$ of $25 \mathrm{~ns}$, the pile-up rate $\mathrm{PR}$ is

$\frac{N_{\pi}(1-f)}{\pi R_{\min }^{2}} \cdot \pi R_{c}^{2} \cdot \Delta T_{\text {cal }}$;

it corresponds to 1.2 event for $R_{c}=2 \mathrm{~cm}$. Once accounting for pile-up, the $\pi \rightarrow e$ misidentification probability

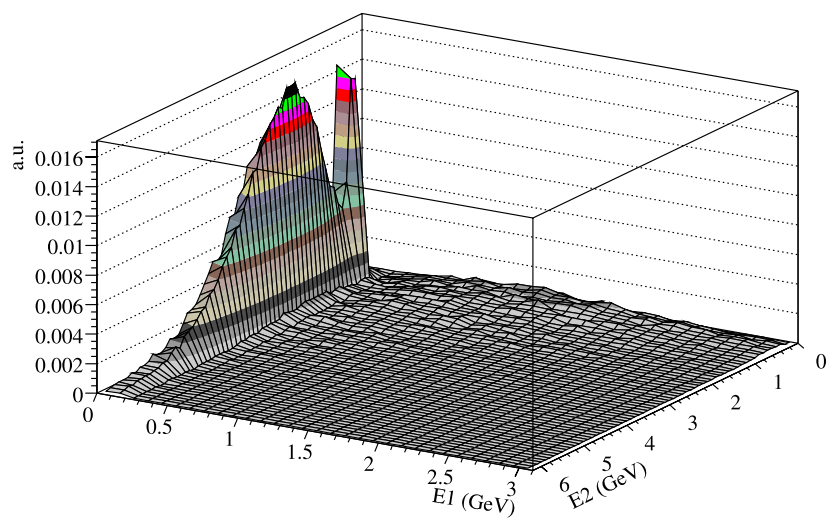

Fig. 7 Energy deposition in the first $10 X_{0}(E 1)$ and in the subsequent $3 \lambda_{I}(E 2)$ along a cylinder of $R_{c}=2 \mathrm{~cm}$ centered at the impact point of undecayed pions in the benchmark beamline grows up to $\sim 10 \%$ at constant detection efficiency for DIF positrons. As a consequence, even if further suppression of the pion background might be achieved exploiting the transverse shower profile or a better longitudinal segmentation, the instrumented beam dump can be fruitfully employed only for moderate beam powers $\left(\sim 10^{9} \pi^{+}\right.$per extraction $)$ or, equivalently, for time resolutions below $1 \mathrm{~ns}$. At larger beam powers, instead of exploiting the instrumentation of the dump, it is more convenient to reduce the angular spread at the entrance of the decay tunnel $(3 \mathrm{mrad}$ in the present case) and, therefore, increase the geometrical acceptance of the scraping taggers at lower $R_{\min }$.

\section{Conclusions}

In the last decade, outstanding progresses have been achieved in the development of fast, radiation hard detectors for tracking and particle identification. Such efforts have been motivated by challenging requests from modern experiments at colliders-firstly the LHC - and from experiments in the field of rare kaon decays. In this paper, we discussed an application of these technologies aimed at tagging $v_{e}$ neutrinos in beams originating from the decay-in-flight of charged pions and based on scraping of secondary and tertiary leptons along the decay tunnel. For a specific beam configuration of moderate power $(\sim 10 \mathrm{~kW}$ for a $14 \%$ duty cycle), we have shown that the tagging system can achieve a suppression of $86 \%$ of the $v_{e}$ background from $K_{e 3}$ decays and of about $60 \%$ of the $v_{e}$ from the decay-in-flight (DIF) of $\mu^{+}$. This setup can also be employed for beams of larger power (up to $\sim 100 \mathrm{~kW}$ at the same duty cycle) without significant loss in performance. At beamlines of a few $\mathrm{kW}$ power, the tagging efficiencies for DIF $v_{e}$ can be further improved $(+16 \%)$ by an additional instrumented beam dump located at the end of the decay tunnel; at larger powers the use of the instrumented dump is limited by the rate of accidentals due to $\pi \rightarrow e$ and $p \rightarrow e$ misidentification.

Acknowledgements We wish to express our gratitude to U. Dore, P. Loverre, M. Mezzetto and F. Ronga for many interesting discussions and a very careful reading of the manuscript. We are grateful to A. Blondel and R. Steerenberg for useful information on the CERNPS. A special thank to R. Felici, whose suggestions speeded up the completion of this work.

Open Access This article is distributed under the terms of the Creative Commons Attribution Noncommercial License which permits any noncommercial use, distribution, and reproduction in any medium, provided the original author(s) and source are credited.

\section{References}

1. L.N. Hand, in Proceedings of Second NAL Summer Study, Aspen, Colorado, 9 Jun-3 Aug 1969, p. 37 
2. B. Pontecorvo, Lett. Nuovo Cimento 25, 257 (1979)

3. I.P. Nedyalkov, Preprint JINR-E1-81-654, 1981

4. L. Nodulman, in Proceedings of 1982 DPF Summer Study on Elementary Particle Physics and Future Facilities (Snowmass 82), Snowmass, Colorado, 28 Jun-16 Jul 1982, p. 574

5. V.V. Ammonosov et al., Proposal SERP-E-152, 1984

6. R.H. Bernstein et al., FERMILAB-Proposal-0788, 1989

7. R.H. Bernstein et al., in Proceedings of the "Meeting on New Directions in Neutrino Physics at FERMILAB", Batavia, IL, USA, 14-16 Sep 1988, FERMILAB-CONF-89-034, 1988

8. L. Ludovici, P. Zucchelli, arXiv:hep-ex/9701007

9. A.A. Aguilar-Arevalo et al. (MiniBooNE Collaboration), Phys. Rev. Lett. 103, 061802 (2009)

10. H. Chen et al. (MicroBooNE Collaboration), FERMILABPROPOSAL-0974, 2007

11. M. Soderberg (MicroBooNE Collaboration), AIP Conf. Proc. 1189, 83 (2009)

12. I. Stancu et al., arXiv:0910.2698 [hep-ex]

13. B. Baibussinov et al., arXiv:0909.0355 [hep-ex]

14. T. Nakaya, PoS NUFACT08, 010 (2008)

15. Y. Itow et al. (The T2K Collaboration), arXiv:hep-ex/0106019

16. D.S. Ayres et al. (NOvA Collaboration), arXiv:hep-ex/0503053

17. D.S. Ayres et al. (NOvA Collaboration), FERMILAB-DESIGN2007-01

18. J.E. Campagne, M. Maltoni, M. Mezzetto, T. Schwetz, J. High Energy Phys. 0704, 003 (2007)

19. V. Barger, M. Dierckxsens, M. Diwan, P. Huber, C. Lewis, D. Marfatia, B. Viren, Phys. Rev. D 74, 073004 (2006)

20. M.V. Diwan et al., Phys. Rev. D 68, 012002 (2003)

21. A.A. Aguilar-Arevalo et al. (MiniBooNE Collaboration), Phys. Rev. Lett. 102, 101802 (2009)

22. P. Huber, M. Mezzetto, T. Schwetz, J. High Energy Phys. 0803, 021 (2008)

23. J.S. Berg et al. (ISS Accelerator Working Group), J. Instrum. 4, P07001 (2009)

24. T. Abe et al. (ISS Detector Working Group), J. Instrum. 4, T05001 (2009)
25. A. Bandyopadhyay et al. (ISS Physics Working Group), Rep. Prog. Phys. 72, 106201 (2009)

26. S.E. Kopp, Phys. Rep. 439, 101 (2007)

27. A.A. Aguilar-Arevalo et al. (MiniBooNE Collaboration), Phys. Rev. D 79, 072002 (2009)

28. A. Ferrari, A. Guglielmi, P.R. Sala, Nucl. Phys. Proc. Suppl. 145, 93 (2005)

29. J.P. Rutherfoord, Nucl. Instrum. Methods A 482, 156 (2002)

30. A. Artamonov et al., J. Instrum. 3, P02010 (2008)

31. C. Civinini, E. Focardi (eds.), Large Scale Applications and Radiation Hardness of Semiconductor Detectors. Proceedings, 8th International Conference, Florence, Italy, June 27-29, 2007, Nucl. Instrum. Methods 596, 1 (2008)

32. G. Anelli et al., CERN-SPSC-P-326, 2005

33. Y. Obayashi et al. (SuperKamiokande Collaboration), in Proceedings of the 31st International Cosmics Ray Conference (ICRC 2009), Lodz, Poland, 7-15 July 2009

34. H.G. Berns, R.J. Wilkes, IEEE Trans. Nucl. Sci. 47, 340 (2000)

35. D.G. Michael et al. (MINOS Collaboration), Nucl. Instrum. Methods A 596, 190 (2008)

36. R. Acquafredda et al. (OPERA Collaboration), J. Instrum. 4, P04018 (2009)

37. M. Guler et al., CERN-SPSC/99-26, 1999

38. J.R. Sanford, C.L. Wang, Empirical formulas for particle production in p-Be collisions between 10 and $35 \mathrm{BeV} / \mathrm{c}$, Brookhaven National Laboratory, AGS internal report, 1967

39. M. Apollonio et al. (HARP Collaboration), Phys. Rev. C 80, 065204 (2009)

40. J.V. Allaby et al., CERN Yellow Report, 70-12, 1970

41. Application Software Group, GEANT Detector Description and tools, June 1993. CERN program library long writeups Q123, version 3.21

42. S. Agostinelli et al., Nucl. Instrum. Methods A 506, 250 (2003)

43. J. Allison et al., IEEE Trans. Nucl. Sci. NS-53, 270 (2006)

44. J.P. Archambault et al., J. Instrum. 3, P02002 (2008)

45. G. Aad et al., J. Instrum. 3, S08003 (2008) 